\title{
THE EXISTENCE OF EFFICIENT LATTICE RULES FOR MULTIDIMENSIONAL NUMERICAL INTEGRATION
}

\author{
HARALD NIEDERREITER
}

Dedicated to Professor Edmund Hlawka on the occasion of his 75th birthday

\begin{abstract}
In this contribution to the theory of lattice rules for multidimensional numerical integration, we first establish bounds for various efficiency measures which lead to the conclusion that in the search for efficient lattice rules one should concentrate on lattice rules with large first invariant. Then we prove an existence theorem for efficient lattice rules of rank 2 with prescribed invariants, which extends an earlier result of the author for lattice rules of rank 1 .
\end{abstract}

\section{INTRODUCTION}

For $s \geq 2$ an $s$-dimensional lattice is the set of all linear combinations with integer coefficients of $s$ linearly independent vectors in $\mathbf{R}^{s}$. We only consider lattices which contain $Z^{s}$ as a sublattice. If $L$ is such a lattice, then $L \cap[0,1)^{s}$ is a finite set consisting, say, of the distinct points $\mathbf{x}_{1}, \ldots, \mathbf{x}_{N}$. The $s$-dimensional lattice rule corresponding to $L$ (or, by a slight abuse of language, the lattice rule $L$ ) approximates the integral $I(f)$ of a function $f$ over $[0,1]^{s}$ by

$$
Q(L ; f)=\frac{1}{N} \sum_{n=1}^{N} f\left(\mathbf{x}_{n}\right) .
$$

We write $X(L)=L \cap[0,1)^{s}=\left\{\mathbf{x}_{1}, \ldots, \mathbf{x}_{N}\right\}$ for the set of nodes in the lattice rule $L$. If we want to emphasize that the number of nodes in a lattice rule is $N$, then we speak of an $N$-point lattice rule. To avoid a trivial case, we always assume that $N \geq 2$.

Lattice rules were originally designed for the numerical integration of periodic functions having $[0,1]^{s}$ as their period interval, and they were introduced by Sloan [15] and Sloan and Kachoyan [16]. Later, the applicability of lattice rules was extended to nonperiodic integrands by Niederreiter and Sloan [14]. A special class of lattice rules has been known for a long time as the method of good lattice points, which goes back to Korobov [5] and Hlawka [3]. We refer to Lyness [9] for a recent survey of lattice rules and to Hua and Wang [4] and Niederreiter $[11,13]$ for expository accounts of the method of good lattice points.

Received April 12, 1990; revised January 17, 1991.

1991 Mathematics Subject Classification. Primary 65D32; Secondary 11K45. 
An important classification of lattice rules was established by Sloan and Lyness [18]. They showed that for any $s$-dimensional lattice rule $L$ there exist a uniquely determined integer $r$ (called the rank) with $1 \leq r \leq s$ and positive integers $n_{1}, \ldots, n_{r}$ (called the invariants) with $n_{i+1} \mid n_{i}$ for $i=1, \ldots, r-1$ and $n_{r}>1$ such that the node set $X(L)$ consists exactly of all fractional parts

$$
\left\{\sum_{i=1}^{r} \frac{k_{i}}{n_{i}} \mathbf{Z}_{i}\right\} \quad \text { with } 1 \leq k_{i} \leq n_{i} \text { for } 1 \leq i \leq r,
$$

and with suitable $\mathbf{z}_{1}, \ldots, \mathbf{z}_{r} \in \mathbf{Z}^{s}$. Here the fractional part $\{\mathbf{t}\}$ of $\mathbf{t}=$ $\left(t_{1}, \ldots, t_{s}\right) \in \mathbf{R}^{s}$ is defined by

$$
\{\mathbf{t}\}=\left(\left\{t_{1}\right\}, \ldots,\left\{t_{s}\right\}\right) \in[0,1)^{s},
$$

where $\{t\}=t-\lfloor t\rfloor$ for $t \in \mathbf{R}$. The points listed in (1) are all distinct, and so the number $N$ of nodes satisfies $N=n_{1} \cdots n_{r}$. The lattice rules that are used in the method of good lattice points are precisely the lattice rules of rank 1.

In the present paper we will, first of all, present evidence that in the search for efficient lattice rules one should concentrate on lattice rules with large first invariant $n_{1}$ (see $\S 2$ ). Then we will prove an existence theorem for lattice rules of rank 2 which shows what kind of efficiency one can expect if the invariants $n_{1}$ and $n_{2}$ are prescribed (see $\$ 3$ ). This theorem can be viewed as an extension of the existence theorem for efficient lattice rules of rank 1 in Niederreiter [12].

To assess the efficiency of lattice rules, we use a standard procedure in numerical integration, namely to consider the order of magnitude of error bounds (for suitable classes of integrands) in terms of the number $N$ of nodes. To describe the most important error bounds, we introduce the following definitions and notations.

Definition 1. The dual lattice $L^{\perp}$ of a lattice $L$ is defined by

$$
L^{\perp}=\left\{\mathbf{h} \in \mathbf{R}^{s}: \mathbf{h} \cdot \mathbf{x} \in \mathbf{Z} \text { for all } \mathbf{x} \in L\right\},
$$

where $\mathbf{h} \cdot \mathbf{x}$ denotes the standard inner product of $\mathbf{h}$ and $\mathbf{x}$.

For a lattice rule $L$ we have $L \supseteq \mathbf{Z}^{s}$, and so it follows that $L^{\perp} \subseteq \mathbf{Z}^{s}$. For $h \in \mathbf{Z}$ we put $r(h)=\max (1,|h|)$, and for $\mathbf{h}=\left(h_{1}, \ldots, h_{s}\right) \in \mathbf{Z}^{s}$ we put $r(\mathbf{h})=\prod_{i=1}^{s} r\left(h_{i}\right)$.

Definition 2. For any lattice rule $L$ and for any real $\alpha>1$ define

$$
R_{\alpha}(L)=\sum_{\substack{\mathbf{h} \in L^{\perp} \\ \mathbf{h} \neq \mathbf{0}}} \frac{1}{r(\mathbf{h})^{\alpha}} .
$$

Now suppose that the integrand $f$ is periodic with period interval $[0,1]^{s}$ and that $f$ is represented by its absolutely convergent Fourier series with Fourier coefficients $\hat{f}(\mathbf{h})$ satisfying $\hat{f}(\mathbf{h})=O\left(r(\mathbf{h})^{-\alpha}\right)$ for some $\alpha>1$. Then Sloan and Kachoyan [17] have shown the error bound

$$
Q(L ; f)-I(f)=O\left(R_{\alpha}(L)\right),
$$

where the implied constant depends only on $f$. Thus, an efficient lattice rule should have a small value of $R_{\alpha}(L)$. To get a criterion independent of $\alpha$, we 
introduce the quantity $R_{1}(L)$. Put

$$
\begin{gathered}
C(N)=\{h \in \mathbf{Z}:-N / 2<h \leq N / 2\}, \quad C^{*}(N)=C(N) \backslash\{0\}, \\
C_{s}(N)=\left\{\left(h_{1}, \ldots, h_{s}\right) \in \mathbf{Z}^{s}: h_{i} \in C(N) \text { for } 1 \leq i \leq s\right\}, \\
C_{s}^{*}(N)=C_{s}(N) \backslash\{\mathbf{0}\} .
\end{gathered}
$$

Definition 3. For any $N$-point lattice rule $L$ let

$$
R_{1}(L)=\sum_{\mathbf{h} \in E(L)} \frac{1}{r(\mathbf{h})},
$$

where $E(L)=C_{s}^{*}(N) \cap L^{\perp}$.

Note that $E(L)$ is nonempty by [14, Proposition 3]. We remark that in the definition of $R_{1}(L)$ we cannot use the same range of summation as in the definition of $R_{\alpha}(L), \alpha>1$, since the resulting infinite series would diverge. The advantage of $R_{1}(L)$ is that all quantities $R_{\alpha}(L), \alpha>1$, can be bounded in terms of $R_{1}(L)$. In fact, in Theorem 1 we will show that $R_{\alpha}(L)=O\left(R_{1}(L)^{\alpha}\right)$ for all $\alpha>1$. Thus, a small value of $R_{1}(L)$ guarantees small values of $R_{\alpha}(L)$ for all $\alpha>1$.

Definition 4. The figure of merit $\rho(L)$ of a lattice rule $L$ is defined by

$$
\rho(L)=\min _{\substack{\mathbf{h} \in L^{\perp} \\ \mathbf{h} \neq 0}} r(\mathbf{h}) .
$$

The quantities $R_{\alpha}(L), \alpha \geq 1$, can be bounded in terms of the figure of merit $\rho(L)$. For $\alpha=1$ we note first that we also have $\rho(L)=\min _{\mathbf{h} \in E(L)} r(\mathbf{h})$ with $E(L)$ as in Definition 3, according to [14, Proposition 1]. Hence, for any $N$-point lattice rule $L$ we have

$$
\frac{1}{\rho(L)} \leq R_{1}(L)=O\left(\frac{(\log N)^{s}}{\rho(L)}\right),
$$

where the upper bound was shown in the proof of [14, Theorem 2]. For $\alpha>1$ we have

$$
\frac{1}{\rho(L)^{\alpha}} \leq R_{\alpha}(L)=O\left(\frac{(1+\log \rho(L))^{s-1}}{\rho(L)^{\alpha}}\right),
$$

where the upper bound was shown in the proof of [17, Theorem 4]. The implied constants in (3) and (4) depend only on $\alpha$ and $s$. An error bound for nonperiodic integrands is based on the following notion.

Definition 5. The discrepancy $D(L)$ of the node set $X(L)$ of an $N$-point lattice rule $L$ is defined by

$$
D(L)=\sup _{J}\left|\frac{\operatorname{card}(X(L) \cap J)}{N}-\operatorname{Vol}(J)\right|,
$$

where the supremum is extended over all half-open subintervals $J$ of $[0,1)^{s}$ of the form $J=\prod_{i=1}^{s}\left[u_{i}, v_{i}\right)$ and where $\operatorname{Vol}(J)$ denotes the volume of $J$.

Now let the integrand $f$ be of bounded variation on $[0,1]^{s}$ in the sense of Hardy and Krause. Then we have

$$
Q(L ; f)-I(f)=O(D(L))
$$


by the Koksma-Hlawka inequality (see [6, Chapter 2]), where the implied constant depends only on $f$. By combining Theorem 1 in [14] and inequality (4) in [14], we obtain

$$
D(L) \leq s / N+\frac{1}{2} R_{1}(L)
$$

for any $N$-point lattice rule $L$. Therefore, a small value of $R_{1}(L)$ guarantees a small discrepancy $D(L)$ and thus a small error bound in (5). The discrepancy $D(L)$ can also be bounded in terms of the figure of merit $\rho(L)$. According to results in [14] we have

$$
\frac{c_{s}}{\rho(L)} \leq D(L) \leq \frac{c_{s}^{\prime}(\log N)^{s}}{\rho(L)}
$$

for any $N$-point lattice rule $L$, where the positive constants $c_{s}$ and $c_{s}^{\prime}$ depend only on $s$.

In view of the results above, we see that an efficient lattice rule $L$ can be characterized as having a small value of $R_{1}(L)$ or a large value of $\rho(L)$, and that these two characterizations are basically equivalent because of (3). For a detailed discussion of various ways of assessing the efficiency of integration rules such as lattice rules we refer to Lyness [8].

\section{SOME SIMPLE BOUNDS}

We show first that the quantities $R_{\alpha}(L), \alpha>1$, can be bounded in terms of $R_{1}(L)$. Let $\zeta(\alpha)=\sum_{h=1}^{\infty} h^{-\alpha}, \alpha>1$, be the Riemann zeta-function.

Theorem 1. For any $N$-point lattice rule $L$ and any $\alpha>1$ we have

$$
R_{\alpha}(L) \leq\left(1+2 \zeta(\alpha) N^{-\alpha}\right)^{s}-1+(1+2 \zeta(\alpha))^{s} R_{1}(L)^{\alpha} .
$$

Proof. By [17, Lemma 1] we have $N \mathbf{x} \in \mathbf{Z}^{s}$ for all $\mathbf{x} \in L$, hence $L^{\perp}$ contains $N Z^{s}$. We write

$$
R_{\alpha}(L)=\sum_{\substack{\mathbf{h} \in N \mathbf{Z}^{s} \\ \mathbf{h} \neq \mathbf{0}}} r(\mathbf{h})^{-\alpha}+\sum_{\mathbf{h} \in L^{\perp} \backslash N \mathbf{Z}^{s}} r(\mathbf{h})^{-\alpha}=: \sum_{1}+\sum_{2} .
$$

Now

$$
\begin{aligned}
\sum_{1} & =\sum_{\mathbf{h} \in N \mathbf{Z}^{s}} r(\mathbf{h})^{-\alpha}-1=\sum_{h_{1}, \ldots, h_{s} \in \mathbf{Z}} r\left(N h_{1}\right)^{-\alpha} \cdots r\left(N h_{s}\right)^{-\alpha}-1 \\
& =\left(\sum_{h \in \mathbf{Z}} r(N h)^{-\alpha}\right)^{s}-1=\left(1+2 \sum_{h=1}^{\infty}(N h)^{-\alpha}\right)^{s}-1 \\
& =\left(1+2 \zeta(\alpha) N^{-\alpha}\right)^{s}-1 .
\end{aligned}
$$

To bound $\sum_{2}$, we use that every $\mathbf{h} \notin N \mathbf{Z}^{s}$ can be uniquely represented in the form $\mathbf{h}=\mathbf{k}+N \mathbf{m}$ with $\mathbf{k} \in C_{s}^{*}(N)$ and $\mathbf{m} \in \mathbf{Z}^{s}$. We have $\mathbf{h} \in L^{\perp}$ if and only if $\mathbf{k} \in L^{\perp}$. Thus, the $\mathbf{h} \in L^{\perp} \backslash N \mathbf{Z}^{s}$ are exactly given by all points $\mathbf{k}+N \mathbf{m}$ with $\mathbf{k} \in E(L)$ and $\mathbf{m} \in \mathbf{Z}^{s}$, where $E(L)$ is as in Definition 3. Therefore,

$$
\sum_{2}=\sum_{\mathbf{m} \in \mathbf{Z}^{s}} \sum_{\mathbf{k} \in E(L)} r(\mathbf{k}+N \mathbf{m})^{-\alpha} .
$$

We claim that

$$
r(\mathbf{k}+N \mathbf{m}) \geq r(\mathbf{k}) r(\mathbf{m}) \quad \text { for } \mathbf{k} \in C_{s}(N), \mathbf{m} \in \mathbf{Z}^{s} .
$$


It suffices to show

$$
r(k+N m) \geq r(k) r(m) \text { for } k \in C(N), m \in \mathbf{Z} .
$$

This inequality is trivial whenever $k m=0$. If $k m \neq 0$, then

$$
\begin{aligned}
r(k+N m) & =|k+N m| \geq N|m|-|k| \geq N|m|-\frac{N}{2} \\
& =\frac{N}{2}(2|m|-1) \geq|k||m|=r(k) r(m) .
\end{aligned}
$$

Thus (8) is proved. Using (8), we get

$$
\begin{aligned}
\sum_{2} & \leq \sum_{\mathbf{m} \in \mathbf{Z}^{s}} \sum_{\mathbf{k} \in E(L)} r(\mathbf{m})^{-\alpha} r(\mathbf{k})^{-\alpha} \\
& =\left(\sum_{\mathbf{m} \in \mathbf{Z}^{s}} r(\mathbf{m})^{-\alpha}\right)\left(\sum_{\mathbf{k} \in E(L)} r(\mathbf{k})^{-\alpha}\right)=(1+2 \zeta(\alpha))^{s} \sum_{\mathbf{k} \in E(L)} r(\mathbf{k})^{-\alpha} \\
& \leq(1+2 \zeta(\alpha))^{s}\left(\sum_{\mathbf{k} \in E(L)} r(\mathbf{k})^{-1}\right)^{\alpha}=(1+2 \zeta(\alpha))^{s} R_{1}(L)^{\alpha} .
\end{aligned}
$$

In view of (7), this establishes the result.

We note that $\left(1+2 \zeta(\alpha) N^{-\alpha}\right)^{s}-1 \leq c(s, \alpha) N^{-\alpha}$ with a constant $c(s, \alpha)$ depending only on $s$ and $\alpha$. Furthermore, we have $R_{1}(L) \geq \rho(L)^{-1} \geq N^{-1}$, where the first inequality is obtained from (3) and the second inequality follows from the bound $\rho(L) \leq N$ shown in [14, Proposition 2]. Hence, Theorem 1 yields $R_{\alpha}(L)=O\left(R_{1}(L)^{\alpha}\right)$ with an implied constant depending only on $s$ and $\alpha$.

We consider now lattice rules of arbitrary rank $r$ and with invariants $n_{1}, \ldots$, $n_{r}$ as described in $\S 1$. Note that $n_{1}$ is the largest invariant. The following result is an improvement on the bound $\rho(L) \leq N=n_{1} \cdots n_{r}$ mentioned above.

Proposition 1. We always have $\rho(L) \leq n_{1}$.

Proof. Since the invariants $n_{2}, \ldots, n_{r}$ are divisors of $n_{1}$, it follows from the description of the node set $X(L)$ in (1) that the coordinates of all points of $L$ are rationals with denominator $n_{1}$. Therefore $L^{\perp}$ contains $n_{1} Z^{s}$. In particular, we have $\mathbf{h}_{0}=\left(n_{1}, 0, \ldots, 0\right) \in L^{\perp}$, hence $\rho(L) \leq r\left(\mathbf{h}_{0}\right)=n_{1}$.

The argument in the proof of Proposition 1 also yields general lower bounds for the quantities $R_{\alpha}(L), \alpha \geq 1$. Here and later on, we use the expression

$$
S(m)=\sum_{h \in C^{*}(m)}|h|^{-1} \quad \text { for integers } m \geq 1,
$$

where for $m=1$ we use the standard convention that an empty sum has the value 0 . The following result is obtained from [12, Lemmas 1 and 2].

Lemma 1. For any $m \geq 1$ we have

$$
S(m)=2 \log m+C+\varepsilon(m) \text { with }|\varepsilon(m)|<4 / m^{2},
$$

where $C=2 \gamma-\log 4=-0.23 \cdots$ with $\gamma$ being the Euler-Mascheroni constant. 
Proposition 2. For any $N$-point lattice rule we have

$$
\begin{gathered}
R_{\alpha}(L) \geq\left(1+2 \zeta(\alpha) n_{1}^{-\alpha}\right)^{s}-1 \text { for } \alpha>1, \\
R_{1}(L) \geq\left(1+\frac{1}{n_{1}} S\left(\frac{N}{n_{1}}\right)\right)^{s}-1 .
\end{gathered}
$$

Proof. We have shown in the proof of Proposition 1 that $L^{\perp} \supseteq n_{1} \mathbf{Z}^{s}$. Thus for $\alpha>1$,

$$
R_{\alpha}(L) \geq \sum_{\substack{\mathbf{h} \in n_{1} \mathbf{Z}^{s} \\ \mathbf{h} \neq \mathbf{0}}} r(\mathbf{h})^{-\alpha}=\sum_{\mathbf{h} \in n_{1} \mathbf{Z}^{s}} r(\mathbf{h})^{-\alpha}-1=\left(1+2 \zeta(\alpha) n_{1}^{-\alpha}\right)^{s}-1,
$$

where the last identity is shown like the formula for $\sum_{1}$ in the proof of Theorem 1. For $\alpha=1$ we note that $E(L) \supseteq C_{s}^{*}(N) \cap\left(n_{1} \mathbf{Z}^{s}\right)$ and that the elements of the latter set are exactly all points $n_{1} \mathbf{h}$ with $\mathbf{h} \in C_{s}^{*}\left(N / n_{1}\right)$. Therefore,

$$
\begin{aligned}
R_{1}(L) & \geq \sum_{\mathbf{h} \in C_{s}^{*}\left(N / n_{1}\right)} r\left(n_{1} \mathbf{h}\right)^{-1}=\sum_{\mathbf{h} \in C_{s}\left(N / n_{1}\right)} r\left(n_{1} \mathbf{h}\right)^{-1}-1 \\
& =\left(\sum_{h \in C\left(N / n_{1}\right)} r\left(n_{1} h\right)^{-1}\right)^{s}-1=\left(1+\frac{1}{n_{1}} \sum_{h \in C^{*}\left(N / n_{1}\right)}|h|^{-1}\right)^{s}-1 \\
& =\left(1+\frac{1}{n_{1}} S\left(\frac{N}{n_{1}}\right)\right)^{s}-1 .
\end{aligned}
$$

It follows from Proposition 2 and Lemma 1 that if factors depending only on $s$ and $\alpha$ are suppressed, then $R_{\alpha}(L)$ is at least of the order of magnitude $n_{1}^{-\alpha}$ for $\alpha>1$ and at least of the order of magnitude $n_{1}^{-1} \log \left(N / n_{1}\right)$ for $\alpha=1$. For the discrepancy $D(L)$ we have the following general lower bound.

Proposition 3. We always have $D(L) \geq 1 / n_{1}$.

Proof. By the proof of Proposition 1, the coordinates of all points of $X(L)$ are rationals with denominator $n_{1}$. For $0<\varepsilon<n_{1}^{-1}$ let $J_{\varepsilon}$ be the interval $\left[\varepsilon, n_{1}^{-1}\right) \times[0,1)^{s-1}$. Then $J_{\varepsilon}$ contains no point of $X(L)$, and so

$$
D(L) \geq\left|\frac{\operatorname{card}\left(X(L) \cap J_{\varepsilon}\right)}{N}-\operatorname{Vol}\left(J_{\varepsilon}\right)\right|=\operatorname{Vol}\left(J_{\varepsilon}\right)=\frac{1}{n_{1}}-\varepsilon .
$$

Letting $\varepsilon \rightarrow 0+$ we get the desired result.

The bounds established in the propositions above basically carry the same information, but the consequences are displayed most clearly by Proposition 1 . To assess the efficiency of a lattice rule, one has to relate the figure of merit $\rho(L)$ to the number $N=n_{1} \cdots n_{r}$ of nodes. For lattice rules of rank $r \geq 2$, Proposition 1 says that the "relative" figure of merit $\rho(L) / N$ satisfies

$$
\frac{\rho(L)}{N} \leq \frac{1}{n_{2} \cdots n_{r}}
$$

We want $\rho(L) / N$ to be as large as possible for an efficient lattice rule, but (10) shows that this becomes more unlikely the larger the invariants $n_{2}, \ldots, n_{r}$. Indeed, (10) suggests that if we want to look for efficient lattice rules of rank $\geq 2$, then our best bet is to consider lattice rules with large first invariant $n_{1}$. In 
particular, we could consider lattice rules of rank 2 with small second invariant $n_{2}$. This is also supported by the results of the explicit search for efficient lattice rules carried out by Sloan and Walsh [20] which yielded lattice rules of precisely this type.

We will now concentrate on lattice rules of rank 2 . In $\S 3$ we establish results which show the existence of lattice rules $L$ of rank 2 for which the quantities $R_{\alpha}(L), \alpha \geq 1$, are small, and these results are the better the smaller the invariant $n_{2}$.

\section{EXISTENCE THEOREMS FOR LATTICE RULES OF RANK 2}

We consider lattice rules which have a useful additional property, namely that of projection regularity. If $L$ is an $s$-dimensional lattice rule with node set $X(L)$, then for $1 \leq d \leq s$ we define $X_{d}(L)$ to be the subset of $[0,1)^{d}$ obtained by retaining only the first $d$ coordinates of each point of $X(L)$. Then $L$ is called projection regular if $\operatorname{card}\left(X_{d}(L)\right)=n_{1} \cdots n_{d}$ for $1 \leq d \leq r$, where $r$ is the rank and $n_{1}, \ldots, n_{r}$ are the invariants of $L$. A characterization of projection-regular lattice rules was given by Sloan and Lyness [19].

For lattice rules of rank 1 a general existence theorem for efficient lattice rules was established in Niederreiter [12]. It was shown that for every dimension $s \geq 2$ and every integer $N \geq 2$ there exists a projection-regular $N$-point lattice rule $L$ of rank 1 with

$$
R_{1}(L)=O\left(N^{-1}(\log N)^{s}\right),
$$

where the implied constant depends only on $s$. This result is in fact best possible since it was proved by Larcher [7] that for any $N$-point lattice rule $L$ of rank $1, R_{1}(L)$ is at least of the order of magnitude $N^{-1}(\log N)^{s}$.

We now establish an analogous existence theorem for lattice rules of rank 2. We recall that for such lattice rules we have two invariants $n_{1}>1$ and $n_{2}>1$ with $n_{2} \mid n_{1}$, and the number $N$ of nodes is given by $N=n_{1} n_{2}$. For a detailed discussion of lattice rules of rank 2, see Lyness and Sloan [10]. We now fix the dimension $s \geq 2$ and the invariants $n_{1}$ and $n_{2}$, and we put

$$
Z_{i}=\left\{z \in \mathbf{Z}: 0 \leq z<n_{i} \text { and } \operatorname{gcd}\left(z, n_{i}\right)=1\right\} \text { for } i=1,2 .
$$

Let $\mathscr{L}=\mathscr{L}\left(s ; n_{1}, n_{2}\right)$ be the family of all $s$-dimensional lattice rules $L$ of rank 2 with prescribed invariants $n_{1}$ and $n_{2}$ for which the node set $X(L)$ consists exactly of all fractional parts

$$
\left\{\frac{k_{1}}{n_{1}} \mathbf{z}_{1}+\frac{k_{2}}{n_{2}} \mathbf{z}_{2}\right\} \quad \text { with } 1 \leq k_{1} \leq n_{1}, 1 \leq k_{2} \leq n_{2}
$$

as in (1), where $\mathbf{z}_{1}$ and $\mathbf{z}_{2}$ have the special form

$$
\mathbf{z}_{1}=\left(z_{1}^{(1)}, \ldots, z_{1}^{(s)}\right), \quad \mathbf{z}_{2}=\left(0, z_{2}^{(2)}, \ldots, z_{2}^{(s)}\right)
$$

with $z_{1}^{(j)} \in Z_{1}, 1 \leq j \leq s$, and $z_{2}^{(j)} \in Z_{2}, 2 \leq j \leq s$. It follows immediately from [19, Theorems 2.1 and 3.3] that every lattice rule $L \in \mathscr{L}$ is projection regular. For each $L \in \mathscr{L}$ the corresponding lattice in $\mathbf{R}^{s}$ consists exactly of all linear combinations with integer coefficients of the vectors

$$
\mathbf{b}_{i}=\frac{1}{n_{i}} \mathbf{z}_{i} \quad \text { for } i=1,2, \quad \mathbf{b}_{i}=\mathbf{e}_{i} \text { for } 3 \leq i \leq s,
$$


where $\mathbf{e}_{i}$ is the unit vector with 1 in the $i$ th coordinate and 0 elsewhere. Let

$$
M(\mathscr{L})=\frac{1}{\operatorname{card}(\mathscr{L})} \sum_{L \in \mathscr{L}} R_{1}(L)
$$

be the average value of $R_{1}(L)$ as $L$ runs through $\mathscr{L}$. Note that $\operatorname{card}(\mathscr{L})=$ $\phi\left(n_{1}\right)^{s} \phi\left(n_{2}\right)^{s-1}$, where $\phi$ is Euler's totient function.

Theorem 2. For every dimension $s \geq 2$ and any prescribed invariants $n_{1}$ and $n_{2}$ we have

$$
M(\mathscr{L})<c_{s}\left(\frac{(\log N)^{s}}{N}+\frac{\log N}{n_{1}}\right)
$$

with a constant $c_{s}$ depending only on $s$. In particular, for every $s \geq 2$ and any $n_{1}$ and $n_{2}$ there exists a projection-regular s-dimensional lattice rule $L$ of rank 2 with invariants $n_{1}$ and $n_{2}$ such that

$$
R_{1}(L)<c_{s}\left(\frac{(\log N)^{s}}{N}+\frac{\log N}{n_{1}}\right) .
$$

Corollary 1. For every $s \geq 2$ and any prescribed invariants $n_{1}$ and $n_{2}$ there exists a projection-regular s-dimensional lattice rule $L$ of rank 2 with invariants $n_{1}$ and $n_{2}$ such that

$$
R_{\alpha}(L)<c(s, \alpha)\left(\frac{(\log N)^{s}}{N}+\frac{\log N}{n_{1}}\right)^{\alpha} \text { for all } \alpha>1,
$$

where the constant $c(s, \alpha)$ depends only on $s$ and $\alpha$.

Proof. This follows from Theorems 1 and 2.

Corollary 2. For every $s \geq 2$ and any prescribed invariants $n_{1}$ and $n_{2}$ there exists a projection-regular s-dimensional lattice rule $L$ of rank 2 with invariants $n_{1}$ and $n_{2}$ such that the discrepancy of the node set satisfies

$$
D(L)<c_{s}\left(\frac{(\log N)^{s}}{N}+\frac{\log N}{n_{1}}\right)
$$

with a constant $c_{s}$ depending only on $s$.

Proof. This follows from (6) and Theorem 2.

These results guarantee the existence of efficient lattice rules provided that the invariant $n_{1}$ is sufficiently large, or equivalently, that the invariant $n_{2}$ is sufficiently small (if lattice rules with the same number $N=n_{1} n_{2}$ of nodes are compared). This is in accordance with a conclusion that was reached in $\S 2$ by different arguments, namely that among lattice rules of rank 2 the most likely candidates for efficient lattice rules are those with small second invariant $n_{2}$. In view of (2) and (5), the bounds in Corollaries 1 and 2 yield information on the error bounds that can be achieved for suitable lattice rules $L \in \mathscr{L}$.

If we consider the order of magnitude of the bound for $R_{1}(L)$ in Theorem 2, then we observe that the first term $N^{-1}(\log N)^{s}$ is the same as the best possible order of magnitude of $R_{1}(L)$ for lattice rules of rank 1 (compare with (11) and the remarks following it). The second term $n_{1}^{-1} \log N$ is nearly best possible since it follows from the remarks after Proposition 2 that $R_{1}(L)$ is at least of the order of magnitude $n_{1}^{-1} \log n_{2}$. If $n_{1} \approx n_{2}$ (i.e., if $n_{1}$ and $n_{2}$ are of 
the same order of magnitude), then the term $n_{1}^{-1} \log N$ is in fact best possible, since we then have $\log N=\log \left(n_{1} n_{2}\right) \approx \log n_{2}^{2} \approx \log n_{2}$. If powers of $\log N$ are ignored, then the bounds for $R_{\alpha}(L), \alpha>1$, and $D(L)$ in Corollaries 1 and 2, respectively, are best possible, since $R_{\alpha}(L)$ and $D(L)$ are at least of the order of magnitude $n_{1}^{-\alpha}$ and $n_{1}^{-1}$, respectively, by results in $\S 2$.

We emphasize that Theorem 2 provides an upper bound for the average value of $R_{1}(L)$ as $L$ runs through the family $\mathscr{L}$. This means that the bound for $R_{1}(L)$ in Theorem 2 is met by "random" choices of $L \in \mathscr{L}$. This has the following practical implication when searching for efficient lattice rules of rank 2 with prescribed invariants: choose lattice rules $L \in \mathscr{L}$ "at random," then there is a good chance that after a reasonably small number of trials a lattice rule can be found for which the bounds in Theorem 2 and Corollaries 1 and 2 hold. For lattice rules of rank 1 this "randomized" search procedure was already suggested by Haber [1].

The rest of the paper, which can be found in the Supplement section of this issue, is devoted to the proof of Theorem 2 . In $\S 4$ we establish some auxiliary results that are needed for the proof, and in $\S 5$ we complete the proof. The basic ideas of the proof would also work for lattice rules of rank $>2$, but the details become exceedingly more complicated.

\section{BIBLIOGRAPHY}

1. S. Haber, Parameters for integrating periodic functions of several variables, Math. Comp. 41 (1983), 115-129.

2. G. H. Hardy and E. M. Wright, An introduction to the theory of numbers, 4th ed., Clarendon Press, Oxford, 1960.

3. E. Hlawka, Zur angenäherten Berechnung mehrfacher Integrale, Monatsh. Math. 66 (1962), 140-151.

4. L. K. Hua and Y. Wang, Applications of number theory to numerical analysis, Springer, Berlin, 1981.

5. N. M. Korobov, The approximate computation of multiple integrals, Dokl. Akad. Nauk SSSR 124 (1959), 1207-1210. (Russian)

6. L. Kuipers and H. Niederreiter, Uniform distribution of sequences, Wiley, New York, 1974.

7. G. Larcher, A best lower bound for good lattice points, Monatsh. Math. 104 (1987), 45-51.

8. J. N. Lyness, Some comments on quadrature rule construction criteria, Numerical Integration III (H. Braß and G. Hämmerlin, eds.), ISNM85, Birkhäuser, Basel, 1988, pp. 117-129.

9. (1989), 405-419.

10. J. N. Lyness and I. H. Sloan, Some properties of rank-2 lattice rules, Math. Comp. 53 (1989), 627-637.

11. H. Niederreiter, Quasi-Monte Carlo methods and pseudo-random numbers, Bull. Amer. Math. Soc. 84 (1978), 957-1041.

12. __ Existence of good lattice points in the sense of Hlawka, Monatsh. Math. 86 (1978), 203-219.

13. __, Quasi-Monte Carlo methods for multidimensional numerical integration, Numerical Integration III (H. Braß and G. Hämmerlin, eds.), ISNM85, Birkhäuser, Basel, 1988, pp. 157-171.

14. H. Niederreiter and I. H. Sloan, Lattice rules for multiple integration and discrepancy, Math. Comp. 54 (1990), 303-312.

15. I. H. Sloan, Lattice methods for multiple integration, J. Comput. Appl. Math. 12/13 (1985), 131-143. 
16. I. H. Sloan and P. J. Kachoyan, Lattices for multiple integration, Proc. Centre Math. Anal. Austral. Nat. Univ., vol. 6, Austral. Nat. Univ., Canberra, 1984, pp. 147-165.

17. __ Lattice methods for multiple integration: theory, error analysis and examples, SIAM J. Numer. Anal. 24 (1987), 116-128.

18. I. H. Sloan and J. N. Lyness, The representation of lattice quadrature rules as multiple sums, Math. Comp. 52 (1989), 81-94.

19. _L Lattice rules: projection regularity and unique representations, Math. Comp. 54 (1990), 649-660.

20. I. H. Sloan and L. Walsh, A computer search of rank-2 lattice rules for multidimensional quadrature, Math. Comp. 54 (1990), 281-302.

Institute for Information Processing, Austrian Academy of Sciences, SonnenfelsGASSE 19, A-1010 VienNa, AUSTRIA

E-mail address: nied@qiinfo.oeaw.ac.at 


\title{
Supplement to \\ THE EXISTENCE OF EFFICIENT LATTICE RULES FOR MULTIDIMENSIONAL NUMERICAL INTEGRATION
}

\author{
HARALD NIEDERREITER
}

This supplement contains the proof of Theorem 2 in the main part of the paper. In $\S 4$ we establish some auxiliary number-theoretic results that are needed for the proof, and in $\S 5$ we complete the proof. The numbering of lemmas and equations is continued from the main part and the references are to the bibliography in the main part.

\section{AUXILIARY RESULTS}

We need various concepts and results from number theory (see [2] as a general reference). Recall that a function $F$ on the set $\mathbf{N}$ of positive integers is called multiplicative if $F(m n)=F(m) F(n)$ whenever $\operatorname{gcd}(m, n)=1$. We write $\sum_{d \mid n}$ for a sum over all positive divisors $d$ of $n \in \mathbf{N}$. If $F$ and $G$ are multiplicative functions, then the summatory function $\sum_{d \mid n} F(d)$ of $F$ and the Dirichlet convolution $\sum_{d \mid n} F(n / d) G(d)$ of $F$ and $G$ are multiplicative functions of $n \in \mathrm{N}$. Let $\mu$ be the Möbius function and note that $\mu$ is multiplicative. From now on we abbreviate $\operatorname{gcd}(m, n)$ by $(m, n)$. The symbol $p$ will always denote a prime number. For $n \in \mathrm{N}$ let $e_{p}(n)$ be the largest exponent such that $p^{\boldsymbol{e}_{p}(n)} \mid n$ (if $p / n$, then $\left.e_{p}(n)=0\right)$.

Lemma 2. For $k, m, n \in \mathrm{N}$ we have

$$
B(k, m, n):=\sum_{d \mid n} \mu\left(\frac{n}{d}\right)(d, k)\left(m, \frac{d}{(d, k)}\right)= \begin{cases}\phi(n) & \text { if } n \mid k m \\ 0 & \text { otherwise. }\end{cases}
$$

Proof. For $d \in \mathrm{N}$ we have

$$
(d, k m)=(d, k)\left(\frac{d}{(d, k)}, \frac{k}{(d, k)} m\right)=(d, k)\left(\frac{d}{(d, k)}, m\right)
$$

since $d /(d, k)$ and $k /(d, k)$ are coprime. Therefore,

$$
B(k, m, n)=\sum_{d \mid n} \mu\left(\frac{n}{d}\right)(d, k m) .
$$

(C) 1992 American Mathematical Society $0025-5718 / 92 \$ 1.00+\$ .25$ per page 

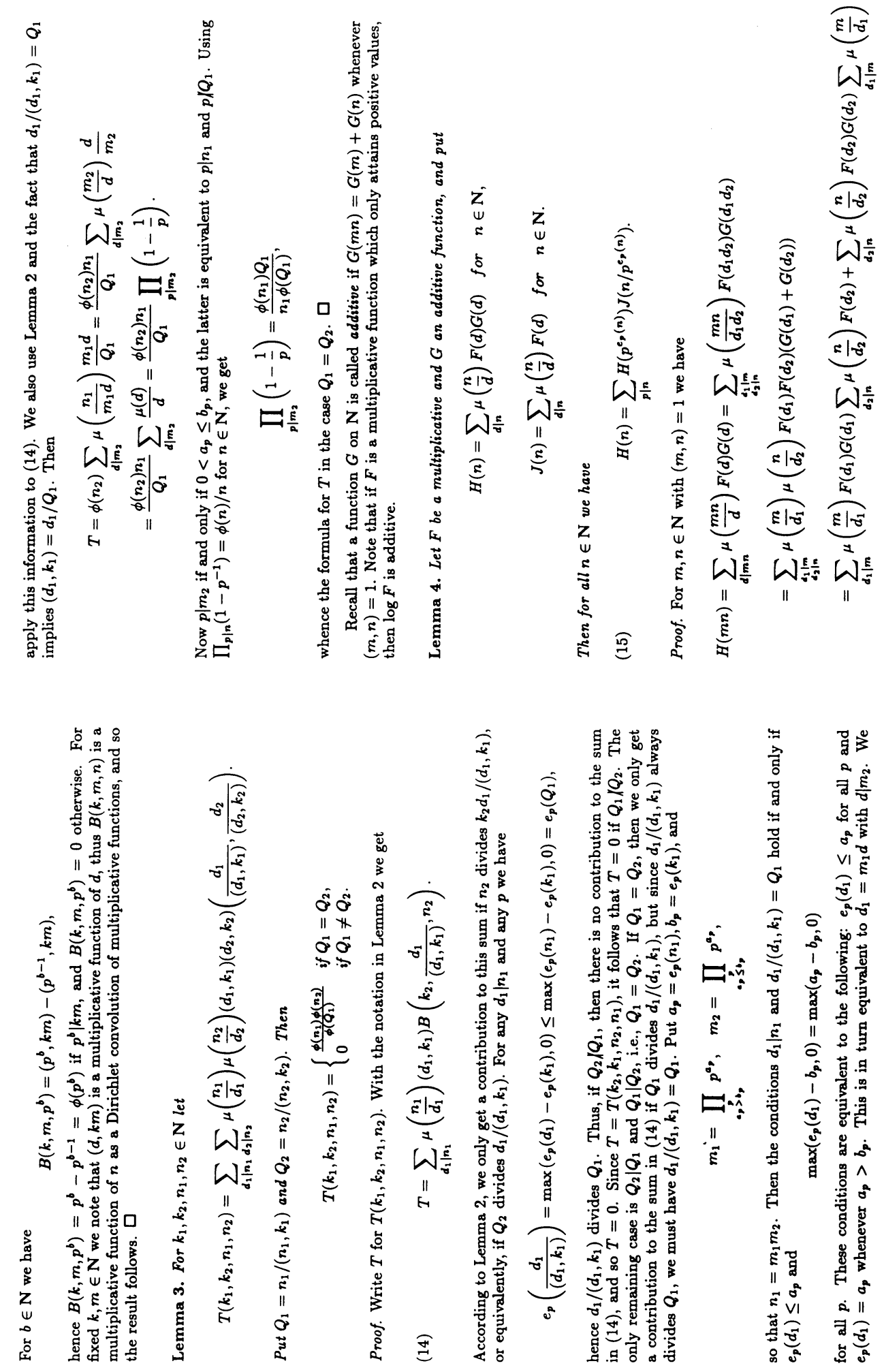

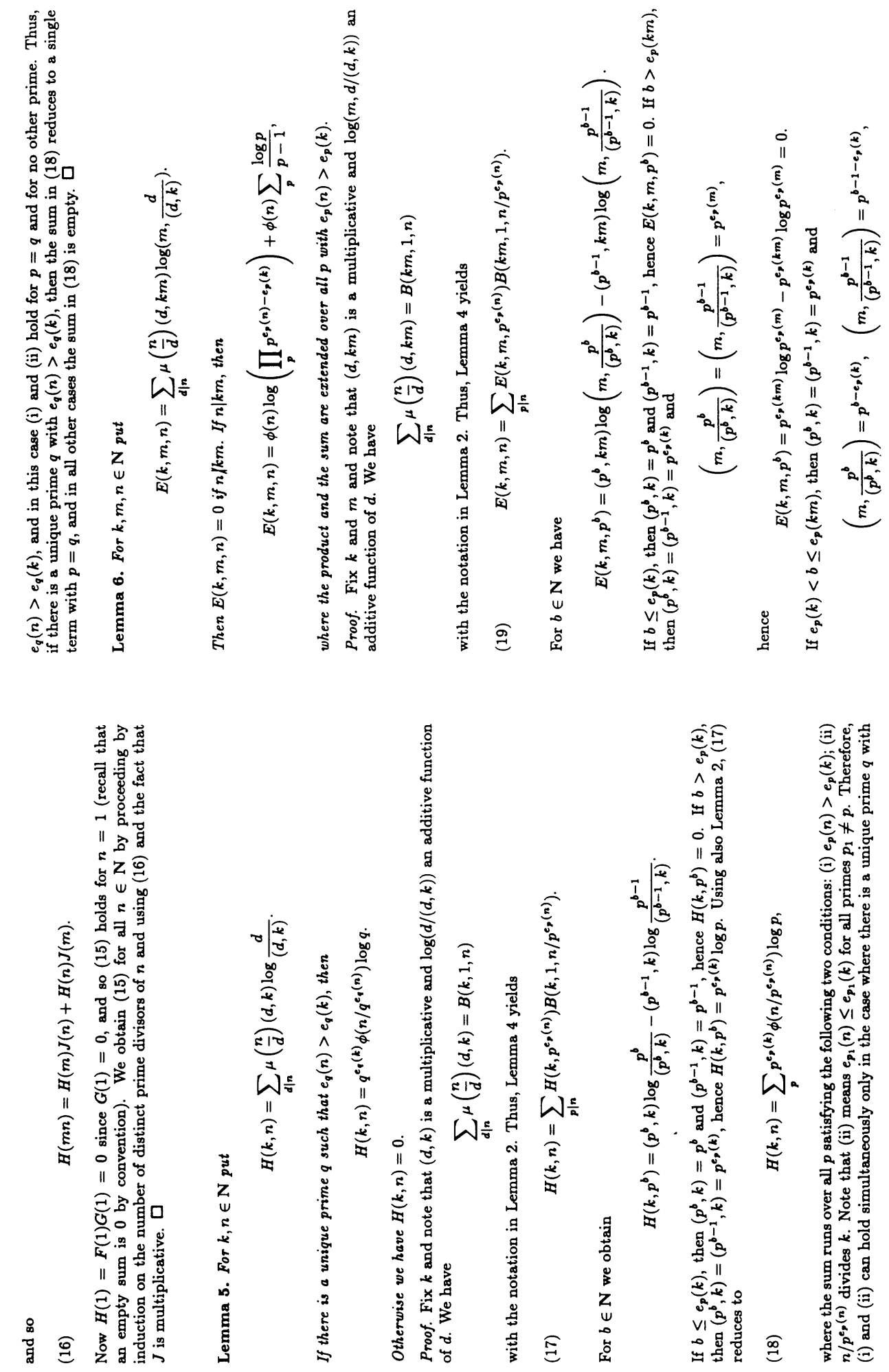

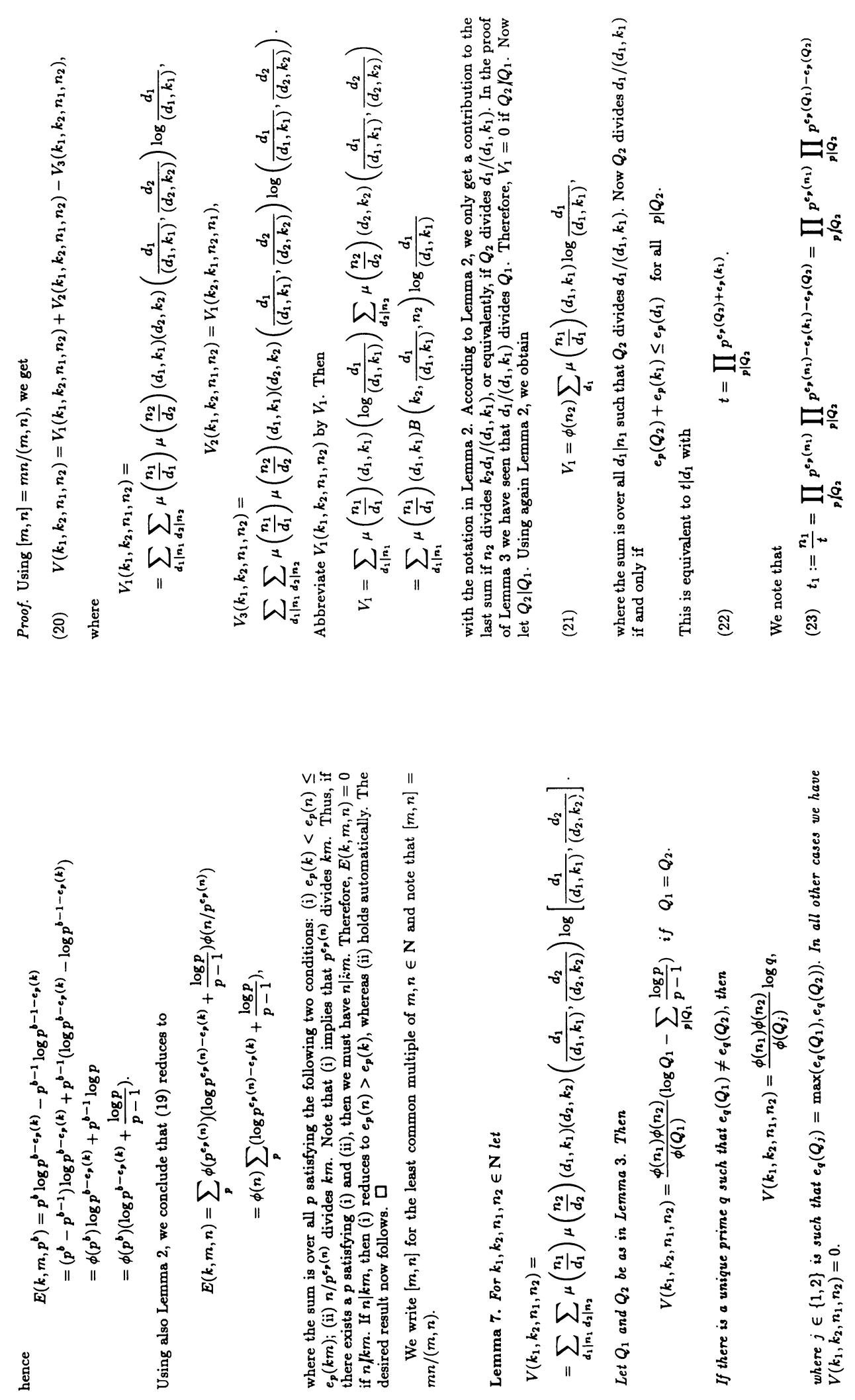


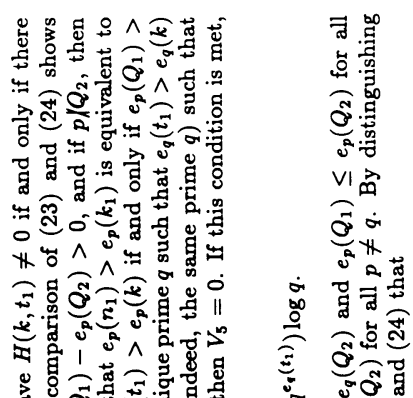

兽

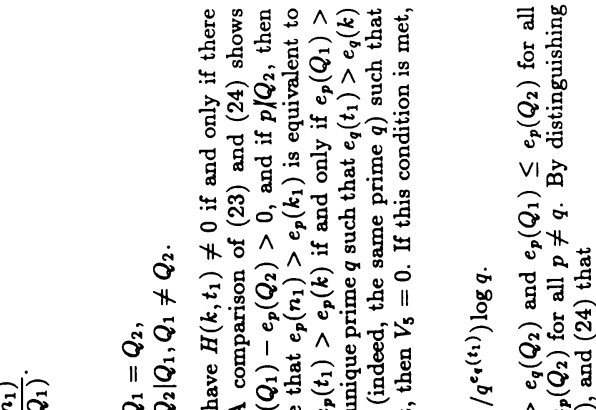

馬

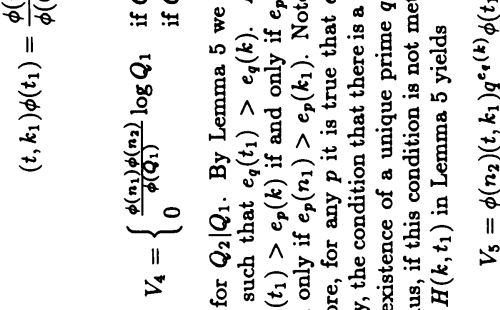

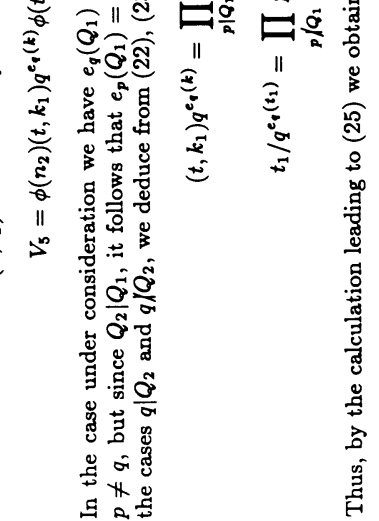
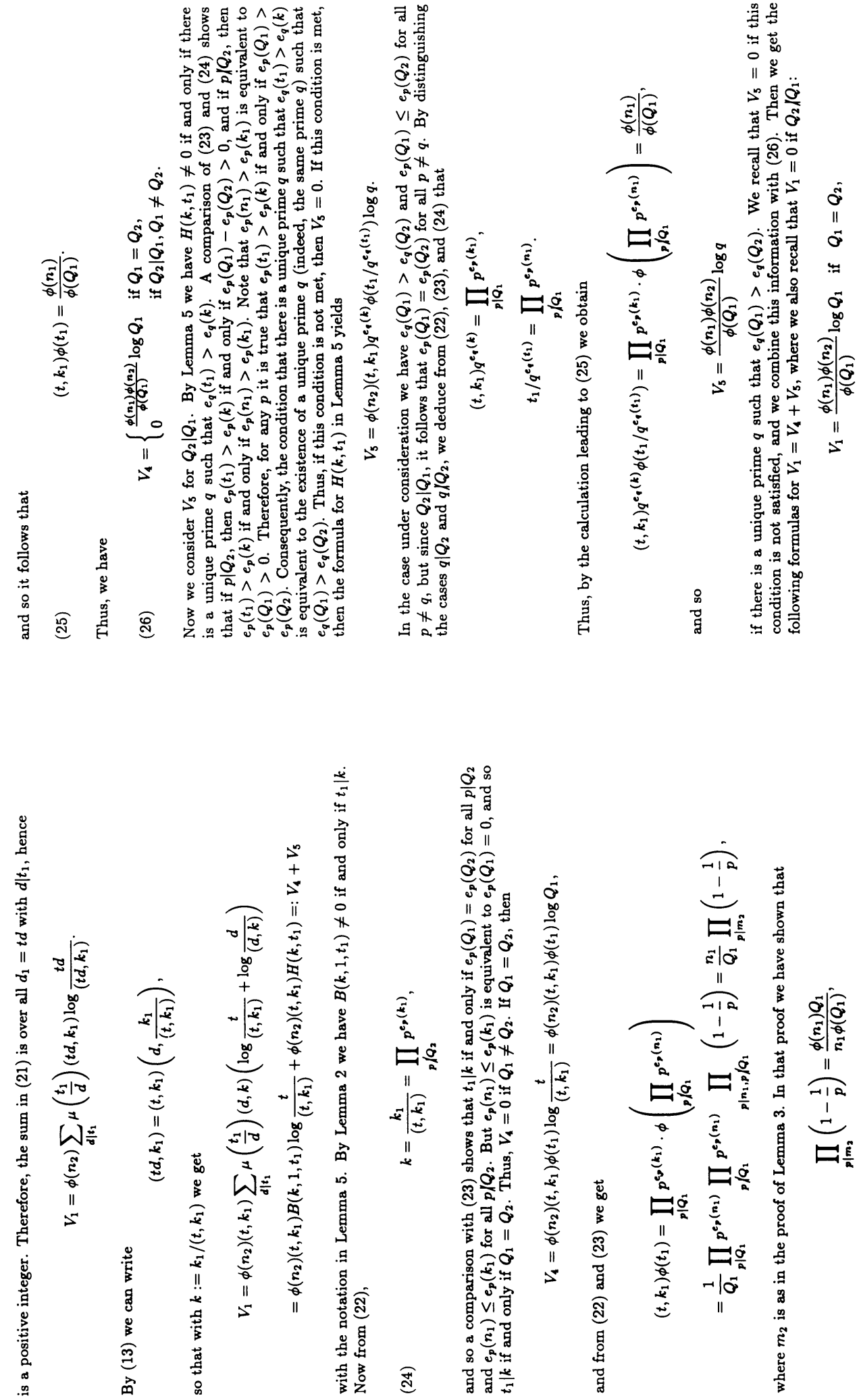

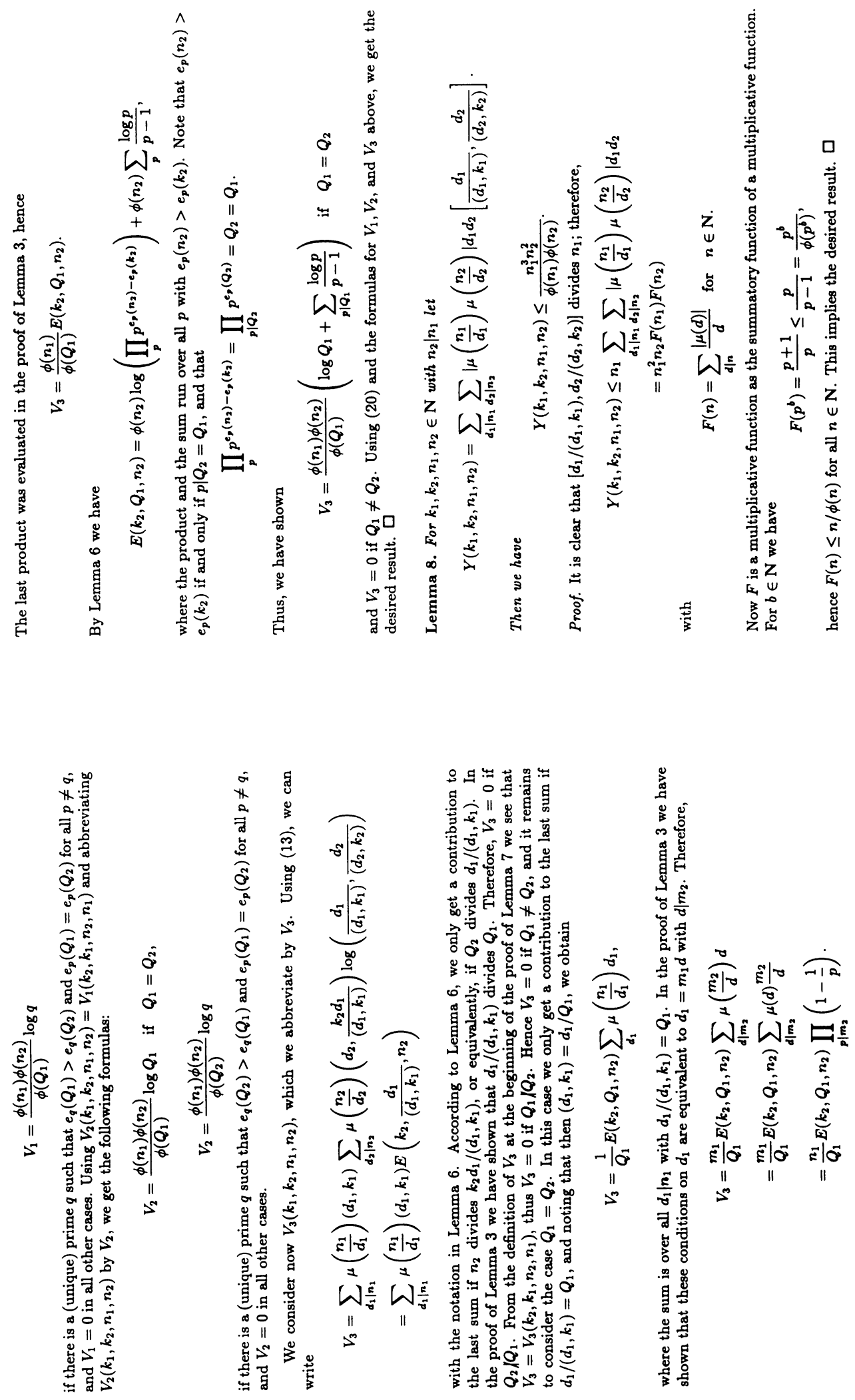

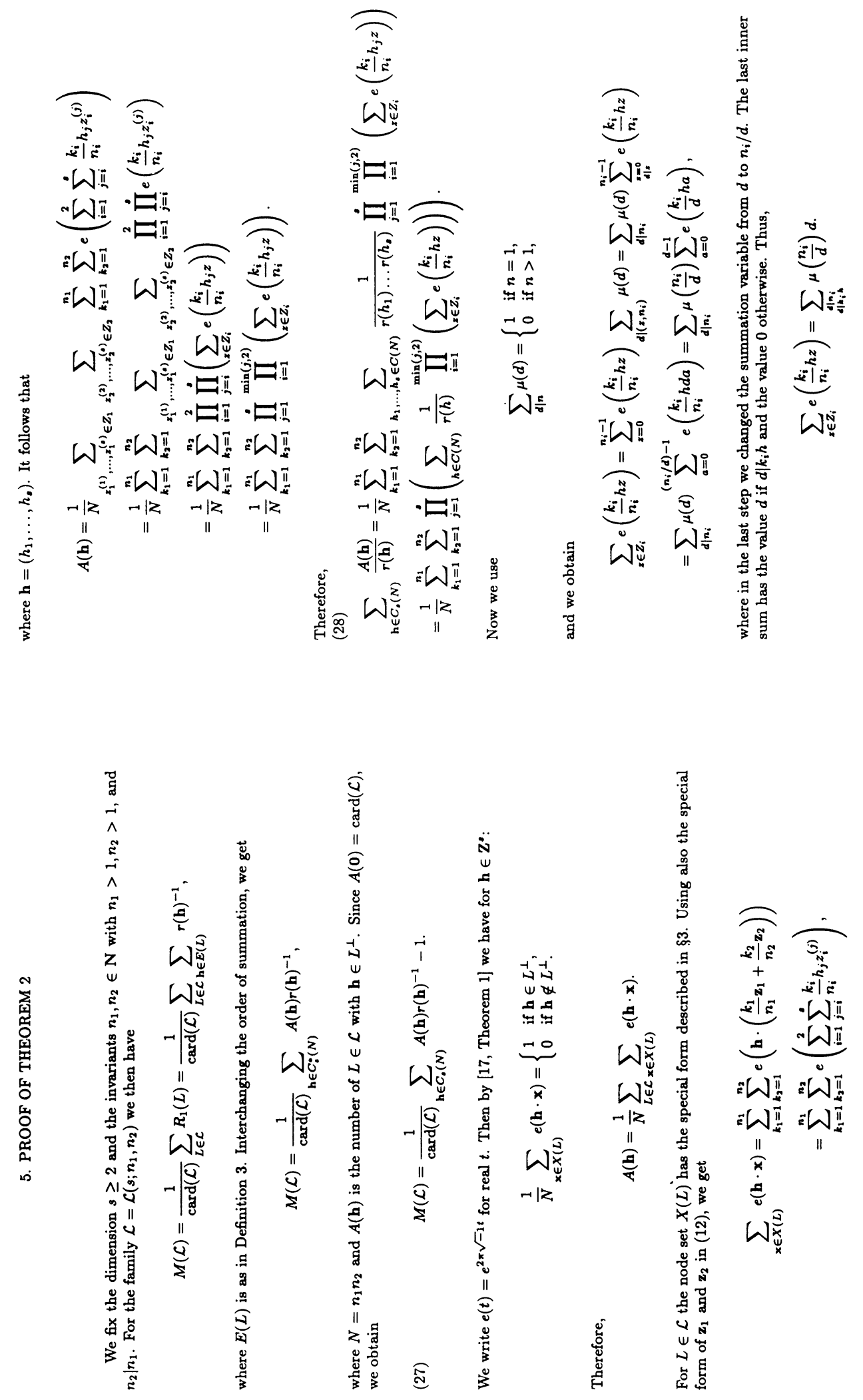

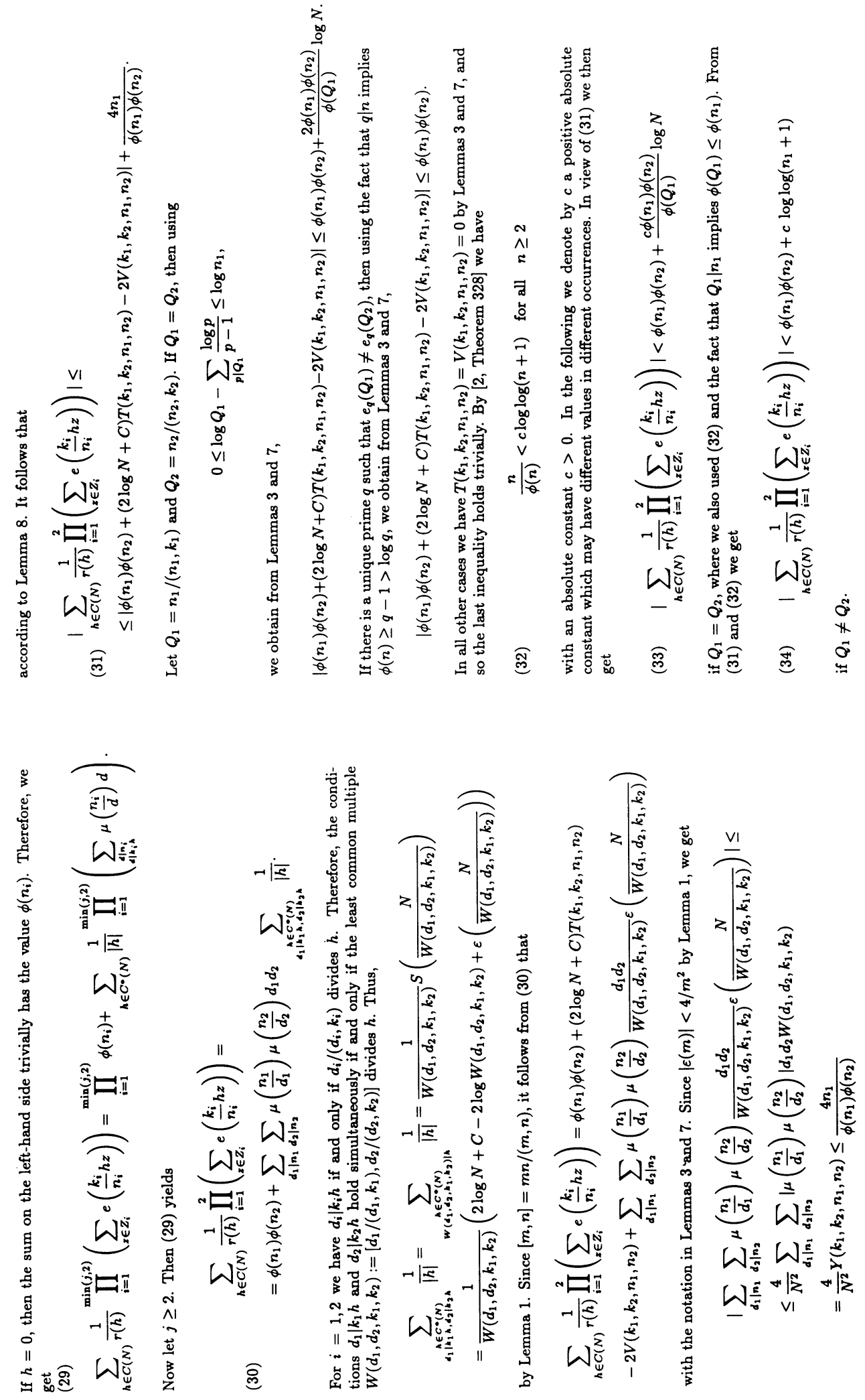

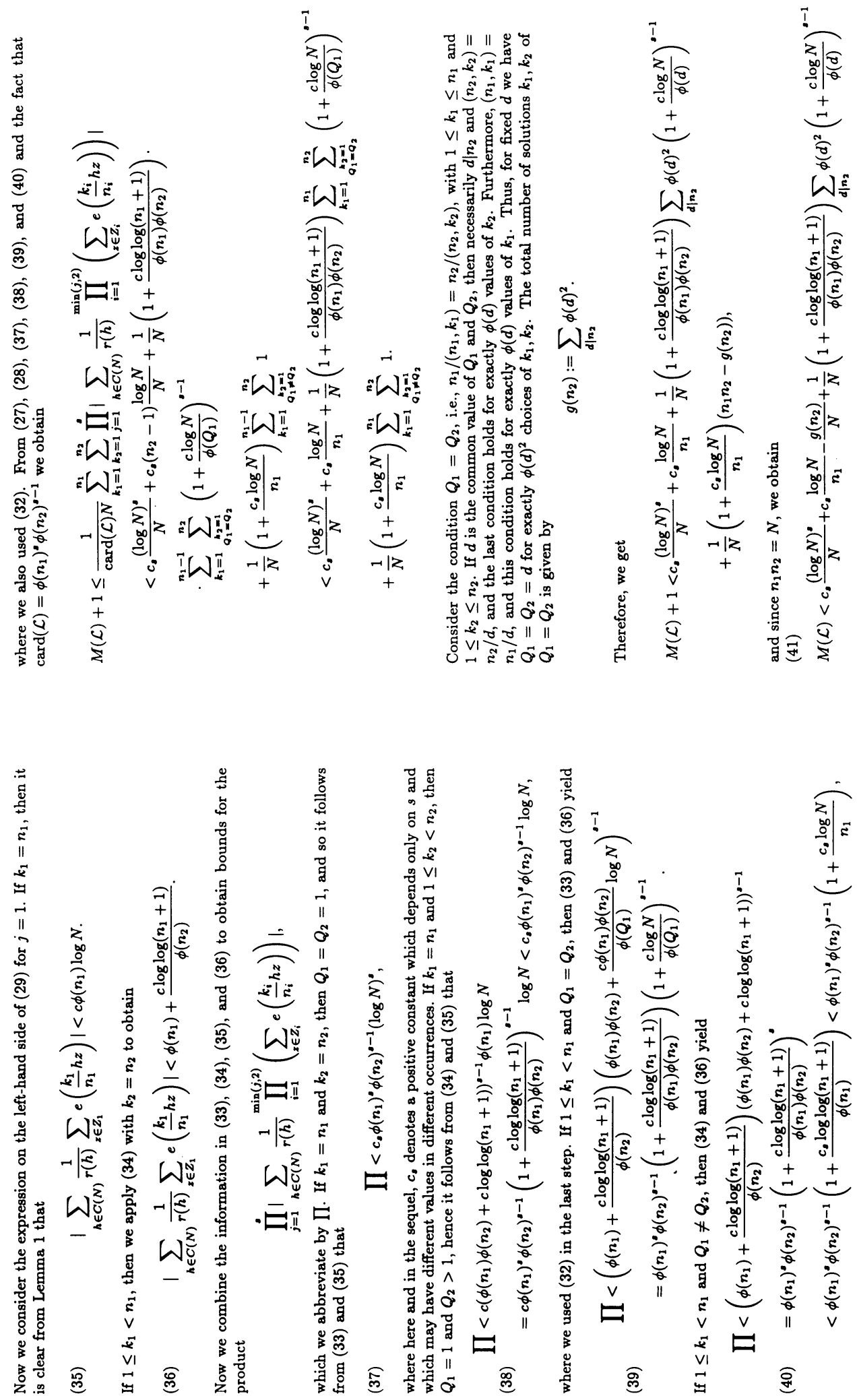


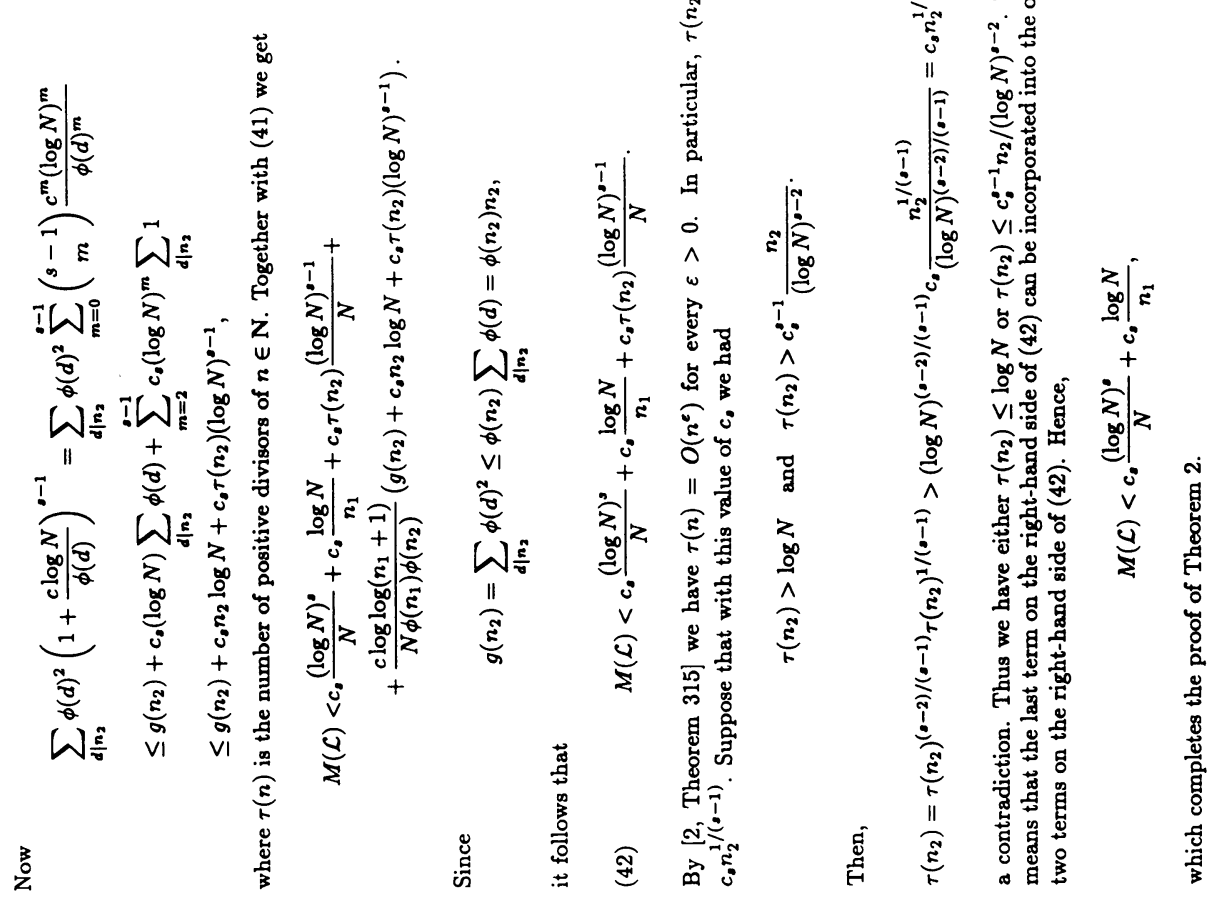

